\title{
Organic Chemistry in the Nineteenth Century : theory of radicals to valency
}

\author{
by Rochelle Forrester \\ Copyright (C) 2020 Rochelle Forrester \\ All Rights Reserved \\ The moral right of the author has been asserted
}

\begin{abstract}
Anyone may reproduce all or any part of this paper without the permission of the author so long as a full acknowledgement of the source of the reproduced material is made.
\end{abstract}

Second Edition

Published 30 March 2020

Preface

This paper was written in order to examine the order of discovery of significant developments in organic chemistry in the nineteenth century. It is part of my efforts to put the study of social and cultural history and social change on a scientific basis capable of rational analysis and understanding. This has resulted in a hard copy book How Change Happens: A Theory of Philosophy of History, Social Change and Cultural Evolution and a website How Change Happens Rochelle Forrester's Social Change, Cultural Evolution and Philosophy of History website. There are also philosophy of history papers such as The Course of History, The Scientific Study of History, Guttman Scale Analysis and its use to explain Cultural Evolution and Social Change and Philosophy of History and papers on Academia.edu, Figshare, Humanities Commons, Mendeley, Open Science Framework, Orcid, Phil Papers, SocArXiv, Social Science Research Network, Vixra and Zenodo websites.

This paper is part of a series on the History of Science and Technology. Other papers in the series are:

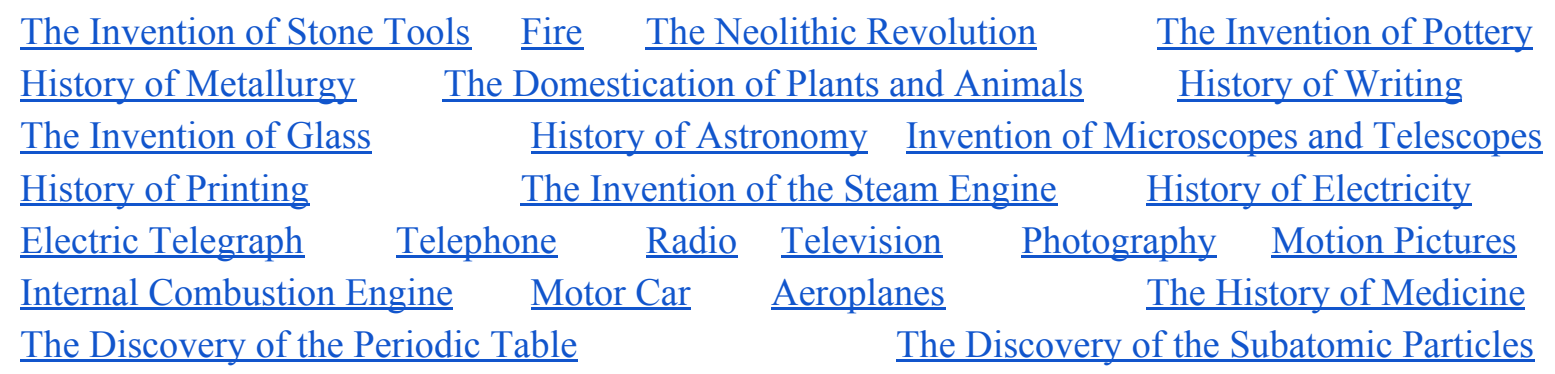

Other papers by Rochelle Forrester include works on Epistemology and the Philosophy of Perception such as Sense Perception and Reality and on quantum mechanics such as the Quantum Measurement Problem and The Bohr and Einstein debate on the meaning of quantum physics. Rochelle Forrester's

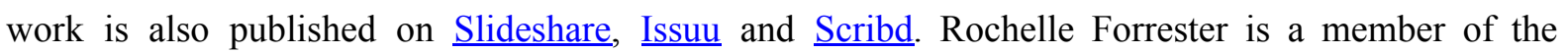
International Network for Theory of History. 
Abstract

There was a specific order of discovery, which was the inevitable path by which our knowledge of organic chemistry was to develop. New methods of analysis of organic materials led to new experimental results, which led to new theories about the nature of organic chemistry. Some of those theories were eventually abandoned, but additional experimental results soon resulted in new theories relating to the valency of carbon atoms and the ability of carbon atoms to combine together to form chains of carbon atoms. Even theories which were later abandoned, due to being contradicted by later experimental results, were logical explanations of knowledge acquired by earlier experimental results. Due to this even incorrect theories can be said to have arisen logically from information available at a particular point in time and to have been an inevitable part of the growth in our knowledge of organic chemistry.

In the early 19th century chemists began to make a distinction between organic chemistry, which concerned materials obtained from animal and plant sources, and inorganic chemistry which dealt with materials from other sources. As the knowledge of organic materials increased, organic chemistry became synonymous with the chemistry of carbon compounds.

Organic analysis at the start of the 19th century was only capable of separating mixtures of related substances and often these procedures resulted in significant chemical alteration of the substances producing misleading results. Lavoisier was the first to develop improved analytic methods for the study of the carbon and hydrogen content of organic materials. He burnt the materials in oxygen or air and weighed the carbon dioxide and water that was formed. Gay-Lussac and Thenard improved the method by reacting the organic material with the oxidizing agent potassium chlorate. The method was further improved when copper oxide replaced the potassium chlorate. The method was still further improved by Berzelius and in 1831 by von Liebig. Liebig's method allowed reliable analysis to take place and was to survive into the 20th century. Methods for the determination of nitrogen in organic substances were devised by Dumas and for the determination of sulphur and halogens by Liebig.

In the early 19th century most chemists believed the products of a living organism were produced through the agency of a vital force present only in living plants and animals. These products could be converted into other products in the laboratory, but could not be created in the laboratory from their elements. This view, known as vitalism, received a serious blow from Wohler in 1828, when he synthesized urea by reacting silver cyanate with ammonium chloride. This however, did not mean the end of vitalism because although Wohler had created an organic product in the laboratory, he had done so by the reaction of two other organic products. Vitalism received a major setback in 1844 when Kolbe 
synthesized acetic acid from non-organic materials, and Berthelot in 1860 showed the possibility of the organic synthesis of organic compounds from the elements carbon, hydrogen, oxygen and nitrogen, leading to the abandonment of vitalism. The development of the concept of the conservation of energy in the middle of the 19th century showed there was no need for the concept of a vital force.

The first attempt to understand the nature of organic compounds was the theory of radicals. Radicals were considered to be the "stable part of a substance that retains its identity through a series of reactions even though it was known to be a compound". Lavoisier considered that when a radical combined with oxygen, an acid was formed. The radical was an element for mineral acids, but was a compound containing both carbon and hydrogen for organic acids. The radicals of different organic acids contained different quantities of carbon and hydrogen. The idea of the radical was extended by experiments by Gay-Lussac on hydrogen cynadide and cyanogen. The cyanide radical was observed to pass unchanged through a series of reactions, so radicals came to be seen as a particularly stable group of atoms that reacted as a unit during chemical reactions.

Support for the radical theory came from the work of Liebig and Wohler in 1832 on benzaldehyde. They converted it to a number of other compounds including benzoyl chloride and benzoic acid and throughout all the changes, the group $\mathrm{C}_{14} \mathrm{H}_{10} \mathrm{O}_{2}$ (actually $\mathrm{C}_{7} \mathrm{H}_{5} \mathrm{O}$ ) remained unchanged. They called this the benzoyl radical. Radicals soon became known as the elements of organic chemistry and chemists began to look for radicals in every compound.

Radical theory however was modified when Dumas studied the reaction of chlorine, bromine and iodine with oil of turpentine and other substances. He considered halogens, such as chlorine, bromine and iodine, were replacing hydrogen within compounds and a given volume of hydrogen was being replaced by the same volume of halogens. He called this discovery the law of substitution. Dumas' work was taken up by Laurent who considered hydrocarbons to be a "fundamental radical", by which "derived radicals"could be obtained by substitution reactions. Derived radicals had similar properties to the fundamental radicals they replaced. Laurents's theory meant radicals could no longer be considered to be an unchangeable group of atoms.

Laurents theory was attacked by Berzelius as it conflicted with his dualistic theory. Berzelius could not see how electro-negative chlorine could replace electro-positive hydrogen, without totally changing the compound. However the experimental evidence, in particular, that involving the chlorination of acetic acid and the studies of the properties of the resulting trichloroacetic acid by Dumas and Melsens, led to an abandonment of the dualistic theory as it applied to organic compounds.

The dualistic theory was opposed by a number of unitary theories proposed by Laurent, Dumas and Gerhardt. Laurent's theory was known as the "nucleus theory", Dumas's as the "theory of types" and Gerhardt's as the "theory of residues". The proliferation of theories reflected the confusion prevailing in organic chemistry in the first half of the 19th century. 
The concept of radicals was enhanced by Bunsen who studied the reactions of a liquid known as cacodyl. He treated cacodyl chloride with zinc and obtained what he considered to be the free cacodyl radical. Later Kolbe isolated what he considered to be the free methyl radical (actually it was ethane) while Frankland believed he had isolated the free ethyl radical (actually butane) by treating ethyl iodine with zinc.

A new type theory was then developed due to the work of Wurtz, Hoffman, Williamson and Gerhardt. Wurtz discovered the primary amines, methyl and ethyl amide. He recognized these compounds were derivatives of ammonia, in which hydrogen had been replaced by methyl or ethyl. Hoffman showed the relationship of the amines to ammonia by alternatively replacing one, then two, then three hydrogen atoms by organic radicals to create primary, secondary and tertiary amines. Hoffman considered all these compounds to belong to an "ammonia type".

Williamson prepared ether by the action of ethyl iodine on potassium ethlate. This meant that a hydrate theory of the structure of alcohols proposed by Liebig was incorrect and Williamson suggested the concept of a water type. This type included compounds such as water, alcohol, ether and methylethyl ether and acetic acids and other acids of that series.

Gerhardt added two further types. These were the hydrogen type that included hydrogen, ethane and butane and the hydrogen chloride type which included hydrogen chloride and ethyl chloride. Gerhardt considered that organic compounds could all be classified as belonging to one of the four types, being the water, hydrogen, hydrogen chloride and ammonia types.

The new type theory was useful as a means of classifying the increasing number of organic compounds but it did little to explain the constitution of the compounds or the arrangement of the atoms in the molecule. The chemical formulae, according to type theory, were reaction formulae. They represented methods of formulation and decomposition, and indicated there were chemical similarities between compounds of the same type. To understand the constitution of the compounds, it was necessary to go beyond the radicals to the atoms, and to look at the arrangement of the atoms in the radical.

Progress to understanding the constitution of compounds and radicals, was made by Frankland. His studies of the combination of organic materials with metals, and other experimental work, showed that an atom of nitrogen, phosphorus, antimony and arsenic always combined with three or five organic radicals. Mercury, oxygen and zinc combined with two organic radicals. Frankland considered "no matter what the character of the uniting atoms may be, the combining power of the attracting element ... is always satisfied by the same number of these atoms". The term "combining power" was eventually replaced by valency. The idea of valency was supported by the law of definite and multiple proportions which implied that atoms had an exact and limited capacity to combine. Valency was also suggested by the experimental observation of substitution. It was known since 1834 that one atom of chlorine replaced one atom of hydrogen, while one atom of oxygen replaced two atoms of hydrogen. Frankland's theory was not immediately accepted, because formulae were 
uncertain and based upon uncertain atomic weights, and because chemists were still imbued with the concepts of radicals and types.

Frankland's work was carried on by Kekule and Couper who simultaneously in 1858 produced improved theories of chemical structure. Their theories brought clarification to ideas that were slowly developing amongst chemists over the previous twenty years. Their ideas had two main themes, the quadrivalency of carbon, and the ability of carbon atoms to join together to form a carbon chain. The linking together of carbon atoms, explained the formation of organic compounds containing many carbon atoms. Their work also showed how the linking together of the atoms of a compound could be shown diagrammatically. The quadrivalency of the carbon atom was later abandoned when subsequent research revealed that in some cases carbon is divalent and trivalent.

The work of Kekule and Couper provided an explanation for the structure of aliphatic compounds as consisting of chains of carbon atoms, but it did not explain aromatic compounds, which always contained at least six carbon atoms in the molecule. The simplest of the aromatic compounds was benzene, which had been discovered by Faraday in 1825 . Kekule suggested the structure of the benzene molecule was a closed ring of six carbon atoms, each of which had a hydrogen atom attached to it. Kekule's theory for the benzene molecule and the nature of aromatic compounds was later to receive experimental confirmation.

The idea of the linking of atoms and the structural representations that were derived from it, form the basis of modern organic chemistry. These theories enabled chemists to understand the relationships between already known compounds, and to discover and create a vast number of new compounds.

The later half of the 19th century saw many more developments in structural chemistry. In 1862 a triple bond was discovered for acetylene and in 1864 a double bond for ethylene. In 1870 Markovnikov's rule was formulated concerning the influence of neighboring groups, on the reactivity of individual parts of organic molecules. Van Hoff and Le Bel provided a picture of the valence bond as uniformly directed in space. The three dimensional view of valence, led to the idea of new types of isomerism, such as cis-trans isomerism.

Analysis of the order of discovery

Organic chemistry began with the development of new and improved methods for organic analysis. The new methods experimental results showing groups of atoms which passed unchanged through a series of reactions, led to the development of the radical theory. The radical theory was forced to be modified when new experimental results showed halogens replacing hydrogen, within organic compounds, leading to the law of substitution. The law of substitution spelt the end of the dualistic theory in relation to organic compounds, and gave rise to a series of theories by Laurent, Gerhardt and Dumas. Eventually the new type theory arose as a means of classifying organic compounds. There is an order of discovery from improved analytic methods, to new experimental discoveries which then resulted in new 
theories to explain the experimental results. This process occurred with both the radical theory and the development of the law of substitution, and the theories created by Laurent, Gerhardt and Dumas which attempted to explain the law of substitution. The development of the new type theory also resulted from experimental results that showed that certain compounds were related to each other.

The same situation is shown by the efforts of chemists to understand the structure of compounds and radicals. Frankland's study of the combining of organic materials with metals, was dependent upon the improved analytic techniques brought into organic chemistry by von Liebig. Frankland's experimental work led to the development of the concept of combining power or valency. Frankland's work was carried on by Kekule and Couper who produced theories concerning the quadrivalency of carbon and the linking together of carbon atoms to form carbon chains. This then led to Kekule's theory of the structure of the benzene molecule and of aromatic compounds. Again one sees new analytic methods leading to experimental results, which then led to the concept of valency, which then led to theories concerning the valency of carbon and to the structure of the benzene molecule.

There was a specific order of discovery which was the inevitable path by which our knowledge of organic chemistry was to develop. Even theories which were later abandoned, due to being contradicted by later experimental results, were logical explanations of knowledge acquired by earlier experimental results. Due to this, even incorrect theories can be said to have arisen logically from information available at a particular point in time, and to have been an inevitable part of the growth in our knowledge of organic chemistry.

Bibliography:

Hudson, J (1992) The History of Chemistry: Chapman \& Hall New York Findlay, A (1965) A Hundred Years of Chemistry (3rd ed): Methuen, London Ihde, A J (1964) The Development of Modern Chemistry: Dover, New York Leicester, H M (1956) The Historical Background of Chemistry: Dover, New York Partington, J R (1961-70) A History of Chemistry: Macmillan, London 